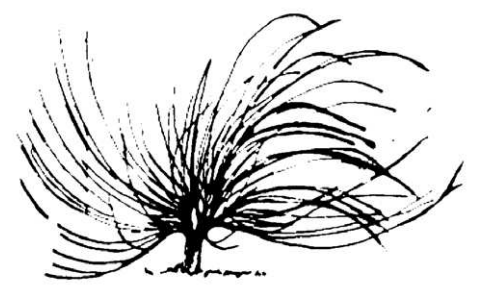

\title{
PLANKTON: una nueva teoría de la organización para el siglo XXI
}

\author{
Javier Simonovich ${ }^{l}$ \\ Max Stern Academia College of Emek \\ Yezreel Valley, Israel \\ javiers@yvc.ac.il
}

\begin{abstract}
Resumen
Las teorías de la organización han sido investigadas y desarrolladas ampliamente durante el siglo XX y siguen siendo de suma importancia en el siglo XXI, explicando las conductas de las organizaciones frente a los desafíos del auge tecnológico.En este trabajo se presentará una reseña histórica de las principales corrientes teóricas de la organización y luego se propondrá una perspectiva innovadora y creativa a través de la pronunciación de la teoría de la organización Plankton, que responde a las nuevas necesidades y desarrollos de las organizaciones de servicios humanos en el mundo tecnológico y global en el que vivimos.La teoría del Plankton organizacional es una perspectíva teórica que ha sido desarrollada por el autor durante los últimos diez años a consecuencia de un intenso trabajo de asesoría a organizaciones de servicios humanos en Israel, y está basada en una extensa experiencia de enseñanza universitaria en la cátedra de Organizaciones de Servicios Humanos en el College Académico del Valle de Yezreel (Emek Yezreel Valley College) en Israel. La teoría de la organización Plankton propone ocho conceptos teóricos por medio de los cuales se aborda la comprensión de la teoría. Los ocho conceptos son:
\end{abstract}

Recibido: 30 de abril de 2013 - Aprobado: 11 de octubre de 2013

1 Decano de Estudiantes y Profesor adjunto (Adjunct Señor Lecturer) Facultad de Servicios Humanos, Max Stern Academia College of Emek Yezreel, Israel. Doctor, Especialista en comportamiento organizacional, desarrollo comunitario, resiliencia y situaciones de crisis y emergencia. Psicoterapeuta de orientación Jungiana. 
planificación, aprendizaje, acabable, normas, conocimiento, técnología, oportunidad y natural.

Palabras clave: teorías de la organización, innovación, administración, planificación

\begin{abstract}
Organization's theories have been widely researched and developed during the twentieth century and remain of paramount importance in the twenty-first century, explaining the behavior of the organizations meet the challenges of the technology boom. In this paper, we present a historical review of the principal theories of the organization and then propose an innovative and creative perspective through the pronunciation of the theory of planktonorganization, responding to new needs and developments of organizations of human services, and to the global technological world in which we live. Plankton Organizational theory is a theoretical perspective that has been developed over the last ten years by the author, as a result of intensive work consulting to human service organizations in Israel, and is based on extensive experience of university teaching in the department of $\mathrm{Hu}-$ man Services Organizations in the Academic College of theYezreel Valley,in Israel. Plankton organization theory proposes eight theoretical concepts through which address the understanding of the theory. The eight concepts are: planning, learning, achievable, norms, knowledge, technology, opportunity and natural.
\end{abstract}

Keywords: organizational theories, innovation, administration, planning

\title{
Introducción
}

T as teorías de la organización han sido investigadas y desarrolladas ampliamente durante el siglo XX y siguen siendo de suma importancia en el siglo XXI, explicando las conductas de las organizaciones frente a los desafíos del auge tecnológico.

En este trabajo se presentará una reseña histórica de las principales corrientes teóricas de la organización y luego se propondrá una perspectiva innovadora y creativa a través de la pronunciación de la 
teoría de la Organización PLANKTON, que responde a las nuevas necesidades y desarrollos de las organizaciones de servicios humanos en el mundo tecnológicoy global en el que vivimos.

La teoría del PLANKTON Organizacional es una perspectiva teórica que ha sido desarrollada durante los últimos diez años a consecuencia de un intenso trabajo de asesoría a organizaciones de servicios humanos, basada en una extensa experiencia de enseñanza universitaria en la cátedra de Organizaciones de Servicios Humanos en el College Académico del Valle de Yezreel (Emek Yezreel Valley College) en Israel.

\section{Las Teorías Organizacionales en el siglo XX}

Las teorías organizacionales se fueron desarrollando durante el siglo XX paralelamente a los procesos de progreso y desarrollo tecnológico, social, político e ideológico que ocurrieron a continuación de la Revolución Industrial del siglo XIX. Las distintas teorías presentadas por diferentes investigadores de la organización estaban basadas en el área de trabajo particular en el cual ellos crecieron y adquirieron su perspectiva profesional y también su filosofía de vida.

Para poder entender en forma ordenada, sistemática y concisa las teorías organizacionales más importantes, su aporte particular y su influencia a través del tiempo, se presentarán las teorías por medio de una taxonomía organizacional (Ver organigrama No.1). Dicha taxonomía está basada en los aportes teóricos y conceptuales de Weiner, $(1990)^{2}$ quien hace una amplia revisión de las distintas teorías que se dividen en teorías clásicas y emergentes.

Las primeras teorías organizacionales que aparecieron a principios del siglo XX son consideradas teorías clásicas.De ellas surgen las teorías emergentes pues son basadas y derivadas de las anteriores.

2 Myron E. Weiner, Profesor Eméritode la Escuela de Trabajo Social, Instituto de Servicios Públicos y en el Programa de Maestría en Relaciones Públicas de la Universidad de Connecticut, USA. Autor de varios libros y artículos sobre el uso de tecnología en administración pública y sin fines de lucro. Como miembro de la WeinerAssociates LLC, Prof. Weiner proporcionó consultoría administrativa y organizacional a organizaciones gubernamentales nacionales e internacionales y sin fines de lucro. 
Figura No.1: Teorías Organizacionales del siglo XX

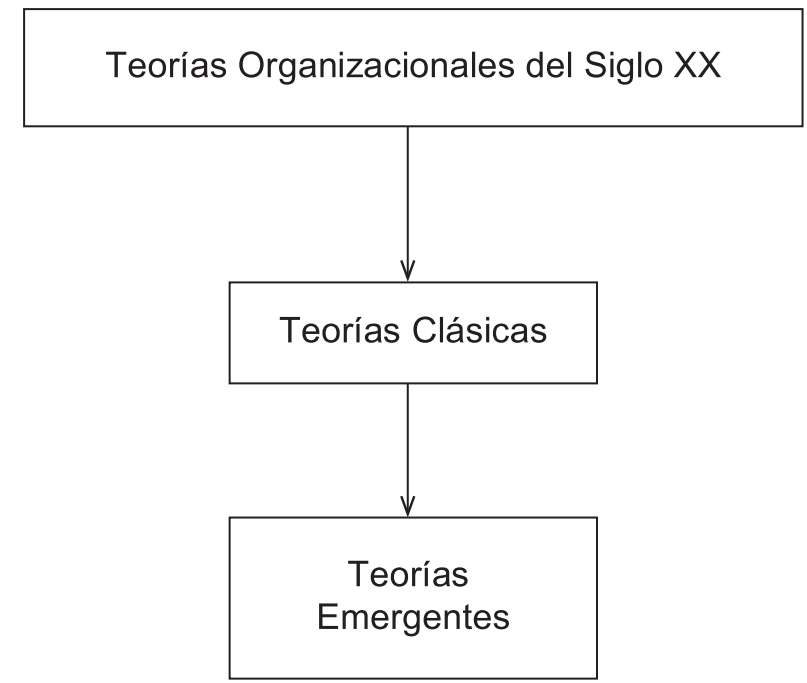

(Adaptación del autor de las teorías presentadas por Weiner, 1990)

Las teorías organizacionales clásicas son aquellas que surgieron a principios del siglo XX. De las teorías clásicas se desprenden desde mediados del siglo XX, las teorías organizacionales emergentes que combinan distintos aspectos de las teorías clásicas y ahondan su especialidad centrándose en algún aspecto particular de las teorías anteriores y en los adelantos tecnológicos y cambios ideológicos que tuvieron lugar luego de la Segunda Guerra Mundial. Con fines pedagógicos y para poder entender la taxonomía de las teorías de la organización, las teorías clásicas son subdivididas en dos subgrupos: a) Teorías Organizacionales Tradicionales; y b) Teorías Sistémicas Tradicionales (Ver Figura No. 2).

\section{Teorías Organizacionales Tradicionales}

Las Teorías Organizacionales Tradicionales comprenden cuatro perspectivas teóricas distintas: 1) Burocrática; 2) Administración Científica; 3) Administración Directiva; y 4) Relaciones Humanas.Por otro lado, las teorías organizacionales sistémicas tradicionales comprenden también cuatro perspectivas teóricas adicionales: 1) Sistémica General; 2) Funcionalismo Estructural; 3) Psicología Organizacional y 4)Sistémica Sociotécnica. 
El modelo burocrático de la organización fue analizado por primera vez por el sociólogo alemán Max Weber (1945), quien describió las cualidades básicas de las organizaciones: la división del trabajo, la jerarquía vertical y constante, definición de normas y regulaciones y canales formales de intercambio de información.

La perspectiva de administración científica fue estudiada cautelosamente en los Estados Unidos por el ingeniero Frederick Winslow Taylor y publicada en 1911 (Taylor, 1945). Taylor propone la maximización de las ganancias por medio de un meticuloso estudio de cuantificación y cálculo de todas las conductas necesarias para poder realizar una tarea. Su perspectiva básica es que los seres humanos están motivados casi exclusivamente por la remuneración económica.

Figura No.2: Teorías Clásicas Sistémicas y Organizacionales Tradicionales

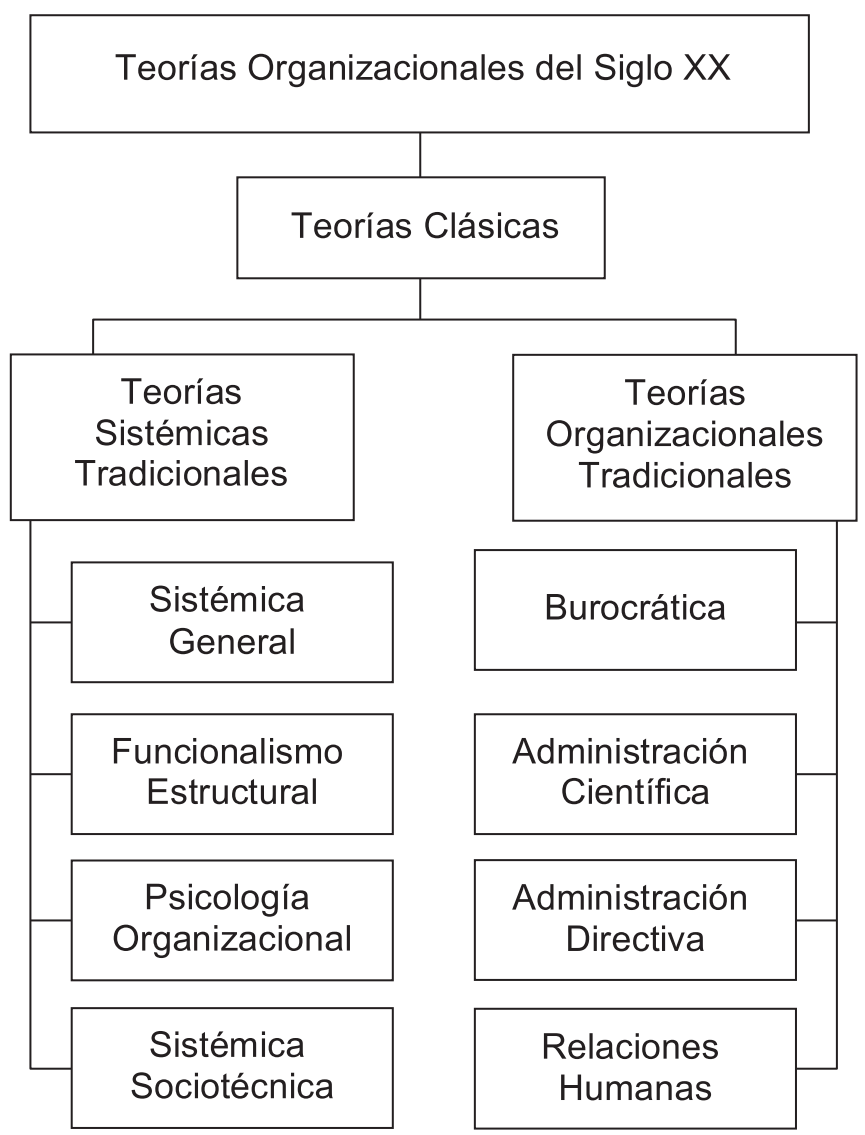

(Adaptación del autor de las teorías presentadas por Weiner, 1990) 
A diferencia de las dos teorías anteriores, teorías cuyos pilares fueron Taylor y Weber, fueron varios los investigadores que contribuyeron a la teoría de la administración directiva en Europa y en los Estados Unidos. Entre ellos algunos de los más destacados fueron Luther Gulieck (el padre de la administración americana) y Lyndall Urwick (respetado teórico de la organización en Gran Bretania). Según la perspectiva de ambos autores, la administración directiva trata de contestar a la pregunta ¿Cuál es la labor del liderazgo ejecutivo en la organización? con el acrónimo POSDECORB que sus letras significan: Planificar, Organizar, entrenamiento del Staff, Dirigir, Coordinar, Reportear a los responsables y preocuparse por el presupuesto (Budget en Inglés) (Gulick \& Urwick, 1937).

La cuarta teoría de las organizacionales tradicionales, la teoría de relaciones humanas, es considerada un movimiento social basado en la perspectiva humanista.Esta perspectiva influyó profundamente a las organizaciones y sociedades humanas tomando en cuenta las necesidades personales y sociales de los individuos que activan en dichas organizaciones, proporcionando la satisfacción de ellas. Por primera vez, en la historia de las organizaciones, los aspectos informales de las relaciones humanas fueron investigados y presentados como importantes componentes en el buen desempeño de los equipos de trabajo. Una de las más destacadas precursoras de las relaciones humanas en las organizaciones fue la trabajadora social y teórica de la filosofía de la administración, Mary Parker Follet (1924), quien puede ser considerada la progenitora de la administración de los servicios humanos en los Estados Unidos y en el mundo.

\section{Teorías Organizacionales Sistémicas Tradicionales}

Las Teorías Organizacionales Sistémicas Tradicionales (TOST) fueron clasificadas por Silverman, (1971) (Ver Figura No. 2). Debe tenerse en claro que la división entre las teorías organizacionales y las teorías sistémicas tradicionales es artificial ya que ambas tratan de entender y analizar la manera en que los seres humanos interrelacionan en grupos y organizaciones en el entorno social. La división es entonces pedagógica para poder entender en forma clara las diferencias y acentos de cada teoría. Las TOST toman de la biología el concepto de sistema compuesto por distintos órganos independientes e interactivos entre sí, 
que se adaptan al entorno existente con el objetivo de sobrevivir y actuar. El punto inicial de análisis será entonces el todo o la perspectiva del sistema y no la de sus partes por separado.

La Teoría Sistémica General (TSG) fue desarrollada por el biólogo Ludwig Von Bertalanffy,(1968) en los años 50 del siglo XX, como un intento de sintetizar la evolución organizacional en una teoría conceptual que contribuye a entender los sistemas humanos. La TSG ayuda a explicar los distintos procesos organizacionales por medio de la interrelación y la interdependencia entre ellos.

Prácticamente, al mismo tiempo que la TSG influyó en el pensamiento social el Funcionalismo Estructural (FE) proporcionó otra perspectiva interesante de las teorías organizacionales. Si bien, la teoría ve a la sociedad y a las organizaciones como un sistema, el enfoque es en cómo las organizaciones e instituciones sobreviven a pesar de los cambios permanentes del entorno natural y social. Talcott Parsons, (1949) uno de los más prominentes teóricos del $\mathrm{FE}$, propuso un paradigma en el que incluye los conceptos de adaptación, logro de objetivos ("goal" en Inglés), integración y latencia (AGIL). El sistema social es visto como una red de subsistemas interrelacionados que funcionan para proveer las necesidades de cada uno de acuerdo con el paradigma de Parsons.

A mediados del siglo XX, surgió una nueva escuela de teorías sistémicas llamada psicología organizacional (TPO) a consecuencia del auge de escuelas psicológicas que fueron creadas entonces. En su base teórica buscaron nuevas técnicas que mejoren el ambiente interno de la organización, que promocionen la aceptación grupal y que mejoren el trabajo por medio de la participación en la toma de decisiones. Entre los más prominentes representantes está Abraham Maslow, (1954) que describió la jerarquía de necesidades de los seres humanos desde las necesidades fisiológicas, la necesidad de seguridad personal, la necesidad de pertenencia y status social, hasta la necesidad de autorrealización. La teoría plantea la existencia de conflictos entre las necesidades de la organización y las necesidades individuales. Estos conflictos, a diferencia de teorías anteriores, se solucionan por medio de la modificación de la estructura organizacional y no por medio de la adaptación del individuo. La situación óptima de una organización es cuando la misma provee los objetivos y las necesidades personales y organizacionales por medio de la participación administrativa. 
Las teorías sistémicas sociotécnicas (TSS) agregaron tres dimensiones al entendimiento de las necesidades humanas: 1) Diferentes entornos organizacionales determinan el comportamiento humano. 2) No existe una única forma de organización apropiada para todas las situaciones. 3) Se debe tener en consideración las estructuras formales y las relaciones informales que existen dentro y fuera de la organización. La perspectiva sociotécnica enfatiza la interrelación entre tecnología, entorno, sentimientos de los miembros de la organización y la estructura organizacional. Ya durante los años 50, Joan Woodward(1958), trabajando en Inglaterra propuso que estructuras y procesos organizacionales, dependen en gran parte de la tecnología existente y del entorno mismo de la organización. Las organizaciones son sistemas abiertos que reaccionan en forma dinámica a las distintas demandas de los cambios tecnológicos.

\section{Teoría Organizacionales Emergentes}

Finalmente, basados en los ricos aportes de las distintas teorías clásicas, surgen desde mediados del siglo XX hasta su culminación, las teorías emergentes que serán presentadas bajo tres corrientes principales:1) las ciencias de la conducta; 2) las ciencias de la administración y 3) las teorías contingentes o situacionales (Ver Figura No. 3). Estas teorías abarcan y profundizan distintos aspectos teóricos y prácticos de las teorías clásicas, agregando y acentuando nuevos elementos y perspectivas científicas innovadoras.

Las teorías emergentes de las ciencias de la conducta se centran en cuatro aspectos: 1) los roles humanos; 2) la dinámica grupal; 3) las relaciones Interorganizacionales y 4) el constante cambio planificado.

La teoría de roles sostiene que cada persona realiza durante su vida un gran número de roles al mismo tiempo o en distintas etapas. Por lo tanto, la teoría de roles es también considerada el punto de encuentro entre la psicología y la sociología. Cada persona cumple una serie de roles de acuerdo con su ubicación y status socio-económico. Estos roles determinan el comportamiento de la persona en las distintas organizaciones y situaciones sociales en las que participa (Merton, 1968). El estudio de los roles no pueden ser separados del estudio de la dinámica grupal. Los seres humanos interaccionamos en pequeños grupos que comprenden una dinámica distintiva. Kurt Lewin, (1948) considerado el padre de la dinámica de grupos,introdujo la perspectiva y la técnica 
de la dinámica de grupos en toda organización bajo el lema "no hay nada más práctico que una buena teoría".

La interdependencia mutua es una de las características de la vida del hombre moderno en las sociedades complejas en las que vivimos. A su vez, se ha desarrollado la interdependencia organizacional donde las organizaciones son mucho menos autónomas y mucho más interdependientes. La teoría de relaciones interorganizacionales (TRI) es característica de sociedades pos-industriales donde existe una economía de servicios, altamente profesional y pluralista. Thompson y McEwen (1958) identificaron cuatro tipos de procesos que gobiernan las relaciones interorganizacionales: la competencia entre organizaciones, la negociación, la cooptación y la creación de coaliciones.

Si el cambio continuo es la única constante en el Universo lo será también en las sociedades y organizaciones en las que vivimos. En el año 1961, el libro La Planificación del Cambio (Bennis et al.) presentó dos tendencias de pensamiento intelectual. La de los sicólogos organizacionales interesados en manejar los procesos de cambio y los conflictos en las organizaciones y la de los planificadores urbanos abogados a la tarea de delinear los parámetros organizacionales de la vida futura. Por lo tanto, la teoría de cambio planificado (TCP) ve la planificación como parte integral del proceso social. Este proceso debe ser racional para poder trazar una política clara, basado en los constantes cambios de las estructuras organizacionales, y finalmente, evaluables y cuantificables.

Figura No. 3: Teorías Organizacionales Emergentes

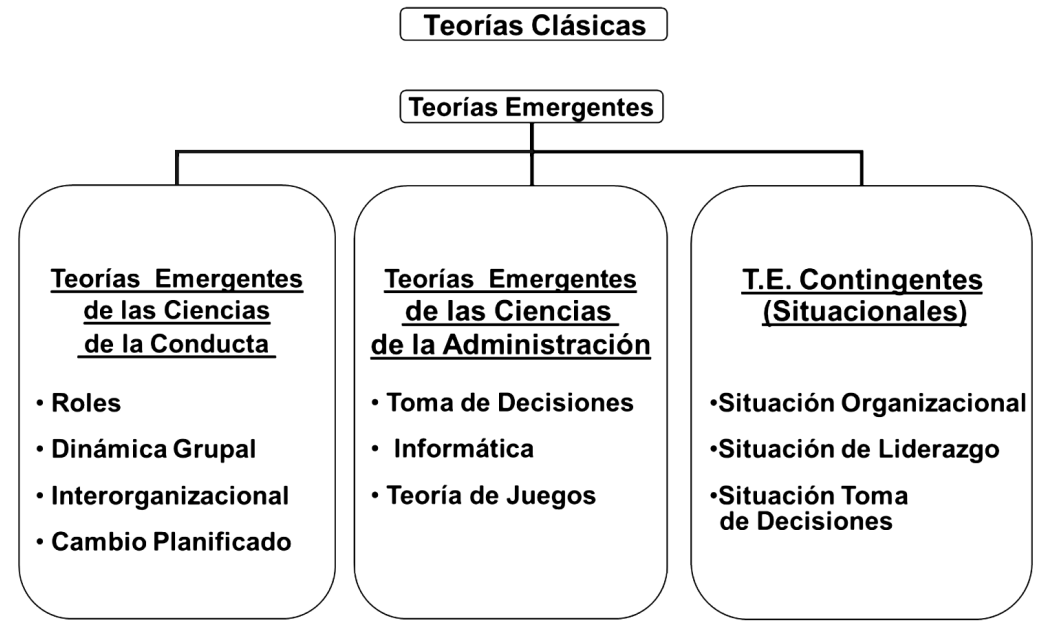

(Adaptación del autor de las teorías presentadas por Weiner, 1990) 
Las teorías emergentes de las ciencias de la administración son muy amplias y a su vez difusas. De todas maneras, pueden ser subcatalogadas de acuerdo con su área de origen y de interés principal. La teoría de toma de decisiones basa su perspectiva en conceptos, propuestas y técnicas que ven a la organización como un ente directivo. Simon (1960) divide las decisiones en dos tipos: decisiones programadas y rutinarias y decisiones inesperadas e intuitivas. El director de la organización debe centrar su tarea en decidir con base en su conocimiento e información. Esta tarea se logra por medio de la teoría de informática y cibernética basada altamente en perspectivas científicas y medibles por medio de las matemáticas.

La teoría de juegos parte de la premisa de que las relaciones entre organizaciones son como juegos de estrategia. Las aplicaciones van de juegos competitivos y guerras hasta juegos económicos o de índole social. Los participantes son considerados "actores" y se encargan de maximizar sus ganancias. John von Neumann y Oskar Morgenstern (1944) publicaron por primera vez la teoría en forma comprehensiva. Desde entonces y hasta hoy se ha convertido en una teoría básica en el entendimiento de las organizaciones y las interrelaciones globales en el mundo.

La teoría emergente contingente (situacional) sostiene que cotidianamente y en forma casual, la organización se enfrenta a distintas situaciones que deben ser resueltas.La primera perspectiva son las situaciones de tipo organizacional donde se debe crear un delicado e interrelacionado diseño de aspectos técnicos y sociales dentro de la organización que deben ser adaptados a las necesidades requeridas por cada situación. La segunda perspectiva son las situaciones de liderazgo organizacional, donde cada director en la organización debe adaptar su estilo de liderazgo a los requisitos específicos de la situación creada, a aquellos a los que debe liderar (incluso a cada uno de sus subordinados). El liderazgo es un proceso dinámico que varía constantemente (Hersey \& Blanchard, 1977). La tercera perspectiva son las situaciones de toma de decisiones en distintos niveles de dirección organizacional que requieren una participación activa en la toma de decisiones por parte del equipo de trabajo.

Hemos presentado hasta aquí una amplia descripción de las principales teorías de la organización desarrolladas durante el siglo XX, las cuales funcionan como infraestructura teórica básica para las nuevas 
propuestas teóricas del siglo XXI. Por lo tanto, pasaremos a presentar la propuesta de la teoría de la organización PLANKTON.

\section{PLANKTON -Teoría de la Organización}

Está claro que las teorías desarrolladas durante el siglo XX no son excluyentes una de otra sino que se las debe considerar complementarias. Cada teoría contribuyó con algunos aspectos que eran de mayor consideración para el lugar y el tiempo en que dicha teoría fue propuesta por el investigador o científico organizacional que la postuló. Es así que la teoría aquí propuesta llamada PLANKTON Organizacional se basa en más de cien años del estudio teórico y práctico de las organizaciones por parte de todos aquellos precursores de las organizaciones que nos antecedieron.

El Plankton es en la naturaleza la unidad básica de la cadena alimenticia en todo el mundo. Si el Plankton desapareciera por alguna catástrofe natural o por una hecatombe creada por la civilización humana, rápidamente desaparecerían de sobre la faz de la tierra casi todas las formas de vida existentes y conocidas hoy. Por eso se considera al Plankton como la base de la cadena ecológica de la vida marina y de la vida terrestre. El Plankton es un nombre general que se le da a los organismos microscópicos que flotan a la deriva de acuerdo con las corrientes marinas en los distintos océanos. El Plankton está compuesto principalmente por algas que obtienen su energía por medio de la fotosíntesis y por protozoarios que se alimentan de partículas y de las mismas algas. Por ello, el Plankton se divide generalmente en fitoplankton (organismos que toman su energía del sol) y zooplankton (organismos que toman su energía de otros organismos).

Dificilmente una persona sin conocimientos científicos y sin instrumentos de alta fidelidad y tecnología pueda visualizar el Plankton. Por su tamaño microscópico es imposible ver las unidades que lo forman a simple vista sin el uso de microscopio. Es igualmente imposible entender la magnitud e influencia del Plankton sobre la vida en la Tierra, sin tomar una perspectiva de miles de kilómetros sobre el mar, preferiblemente con aviones o satélites que circunvalan el planeta Tie-

rra desde la estratosfera. Curiosamente el ser vivo de mayor tamaño hoy en día es el rorcual azul (más conocido como ballena azul) que mide entre 24 y 27 metros y pesa entre 100 y 120 toneladas, se alimenta casi 
exclusivamente de plakton (krill, zooplankton y fitoplankton). Todos los seres vivos en el planeta continúan la cadena alimenticia basada en el plankton. Por lo tanto, el Plankton es la base de la vida en el mundo como lo conocemos hoy.

La teoría de la organización Plankton toma las iniciales de la palabra PLANKTON como acrónimo básico. De esta manera, cada letra genera un concepto profesional a ser aplicado en la comprensión de las organizaciones de servicios humanos hoy. La palabra Plankton es tomada en forma metafórica para poder explicar los componentes básicos, elementales y necesarios en una organización moderna aplicando las cualidades del Plankton antes descriptas.

Así como el Plankton es la base de la vida, cada organización debe poder definir su propio Plankton o unidad básica y mínima de vida que permite el desempeño organizacional, sin el cual la organización perecedería. Cada organización debe poder comprender la conducta dinámica creada por la actividad organizativa interna y su influencia sobre el entorno interorganizacional. La identificación de ese componente mínimo y básico no es tarea fácil. Hace falta para ello una amplia gama de conocimientos teóricos de las organizaciones y un profundo análisis de la organización específica a la que nos referimos. Este proceso es paralelo a la contemplación científica del Plankton por medio del microscopio (análisis y descripción de la unidad organizacional) y a la observación en perspectiva desde la atmósfera (análisis y descripción de la dinámica organizacional general).

Para poder abordar la teoría de la organización Plankton es necesario entonces escribir el acrónimo PLANKTON en forma tal que se pueda enumerar cada letra con la inicial del concepto teórico al que se refiere. Debido a que esta perspectiva teórica fue desarrollada en Israel, donde no se habla español sino hebreo, pero el material bibliográfico está casi totalmente basado en fuentes americanas y europeas, los conceptos del acrónimo están en Inglés. Al lado de cada concepto en inglés, se presenta el concepto con su tentativa traducción al español que no siempre es congruente con el vocablo inglés. 
Tabla No.1: Acrónimo Plankton y sus conceptos

\begin{tabular}{|l|l|l|}
\hline & Ingles & Español \\
\hline $\mathbf{P}$ & Planning & Planificación, proyectar, planear \\
$\mathbf{L}$ & Learning & Aprendizaje, saber, ciencia, sabiduría \\
$\mathbf{A}$ & Achievable & Acabable, factible, ejecutable, se puede lograr \\
$\mathbf{N}$ & Norms & Normas, modelo, regla, unidad típica de \\
$\mathbf{K}$ & Knowledge & Conocimientos, ciencia, destreza, pericia \\
$\mathbf{T}$ & Technology & Tecnología \\
$\mathbf{O}$ & Oportunity & Oportunidad, oportuno, conveniente \\
$\mathbf{N}$ & Natural & Natural, sencillo, genuino, espontaneo \\
\hline
\end{tabular}

(Adaptación del autor)

La teoría de la organización PLANKTON sugiere que el "PLAN da el TON" (en inglés: plan-tone) o que el plan correctamente trazado genera el tono, sonido o resultado de la organización y su correcto desempeño. Una organización PLANKTON es entonces, aquella que en todos sus aspectos indentifica los componentes de cada uno de los conceptos y los puede describir desde su mínima expresión indispensable hasta su máxima manifestación e influencia en el entorno interorganizacional. Presentaremos a continuación los conceptos referidos.

\section{Planificación}

La planificación en una organización de servicios humanos es el instrumento básico que demarca el rumbo y la forma de llevar a cabo la tarea de la organización. El área de planificación de proyectos ha sido profesionalizado a tal punto que existen especialistas en planificación y realización de proyectos. Por eso aquellos que son responsables de dirigir la organización, deben tener substanciales conocimientos y experiencia en planificación de proyectos.

La pirámide de la planificación (Ver Figura No.4) nos ayuda a entender el proceso organizacional que comienza en la planificación, se concretiza en la realización del trabajo a realizar y se culmina con una evaluación de lo realizado de acuerdo con lo planificado. Al terminar este ciclo se comienza nuevamente un nuevo proceso de planificación 
basado en lo aprendido gracias a la evaluación que retroalimenta el proceso de crecimiento de la organización.

Como primera tarea en una organización existente o frente a un nuevo proyecto, se debe fomentar el proceso de pensamiento creativo dentro de la organización (Ver Figura no. 4). Este proceso lleva relativamente menos tiempo que todas las otras etapas pero es de fundamental importancia. La representación gráfica de la cúspide piramidal infiere que por tener la menor superficie toma menos tiempo que las otras etapas del proceso general. Por otro lado, a diferencia de la construcción tridimensional de una pirámide real, este proceso comienza suspendido en el aire, donde la imaginación y el pensamiento creativo son el mejor soporte para un comienzo original e innovador.

Este proceso de planificación irá disminuyendo su altitud y aumentando su superficie hasta alcanzar la base de la pirámide donde se centra la acción a llevarse a cabo por la organización, o sea, que mientras más abstracta la tarea menos tiempo lleva y al llegar a la base, el máximo de tiempo será utilizado en la realización y concretización de la actividad organizacional. 
Figura No.4: Pirámide de planificación
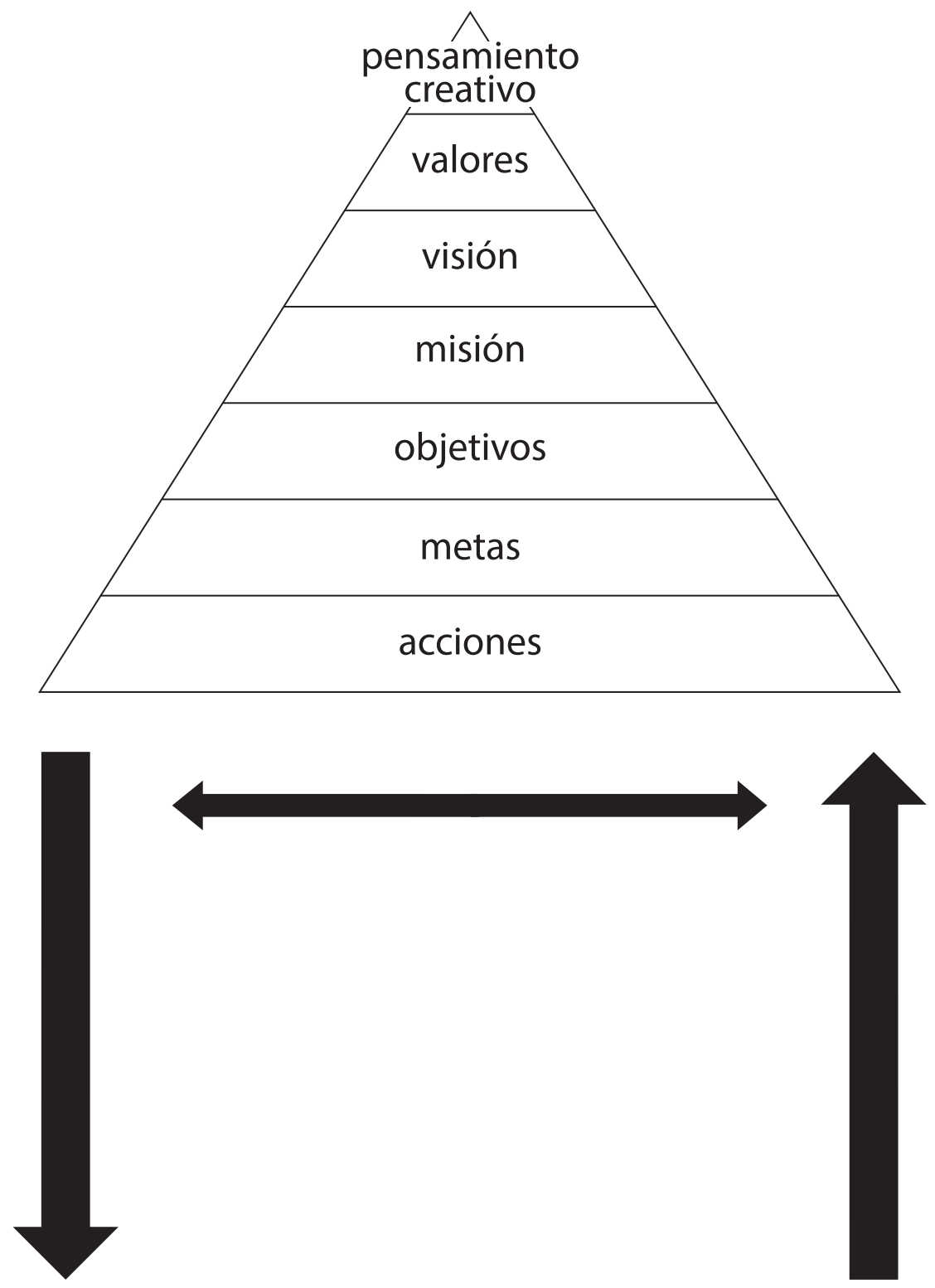

(Adaptación del autor)

Luego de realizar el proceso de pensamiento creativo, y muchas veces simultáneamente la organización debe definir los valores compartidos por los miembros de la organización y conductas esperadas que corresponden y se desprenden de estos valores. Los valores son generalmente el marco ideológico en el que se basa la actividad de la 
organización. Entre los valores más comunes podemos citar justicia, verdad, seguridad, transparencia, servicio, puntualidad y profesionalidad. Luego de haber designado los valores comunes de la organización, se los utiliza para redactar la visión y la misión de la organización. En una organización universitaria, por ejemplo, los valores de educación, conocimiento e igualdad pueden enmarcar los contenidos a ser desarrollados como visión y misión de dicha institución.

La visión de la organización está definida como una frase que incluye cada uno de los valores y denota en forma abstracta una narrativa del punto de máximo éxito que la organización tiende a lograr. Cada organización deberá comprender la esencia de su propia visión y misión. Por ejemplo, la visión de una universidad podría ser redactada como: "La universidad tiende a lograr la mejor educación posible brindando los más amplios conocimientos a sus alumnos, como la mejor forma de fomentar la igualdad de posibilidades entre los ciudadanos". Por ende, la misión de la universidad será capacitar a docentes y alumnos, proporcionándoles las infraestructuras necesarias para la investigación científica con el fin de acercarse cada día lo más posible a la aspirada y deseada visión.

Una vez redactadas la visión y la misión se podrá derivar de ellas los objetivos deseados. La definición de objetivos organizacionales es una tarea que comprende recursos teóricos y prácticos. Los objetivos deben ser redactados por medio de conceptos abstractos que no son cuantificables. El sujeto del objetivo debe crear una causalidad y comportamiento de cambio de una situación a otra determinando el rumbo de acción pero no la meta. El objetivo de la universidad será entonces: capacitar a jóvenes estudiantes en áreas académicas, profesionales y tecnológicas que les brinden una satisfactoria y remunerable inserción en el mercado laboral. Esta definición es clara pero al mismo tiempo abstracta y no incluye parámetros cuantificables.

De cada objetivo deberán recortarse las metas operacionales a lograr por la organización. La diferencia básica entre objetivo y meta es que las metas deben ser obligatoriamente medibles y cuantificables y de aquí su operacionalidad. Si una meta no puede ser medida, no es una meta, y no tiene sentido de ser. Las unidades de medición pueden ser de tiempo, de peso, de longitud, de altura, de nivel de conocimiento, puntaje en un examen, cuantificación de conductas etc. Es imprescindible que para cada objetivo exista por lo menos una meta. Un objetivo 
sin meta no tiene sentido de ser. Volviendo al ejemplo de la universidad, los estudiantes en la universidad pueden definir su meta como: lograr finalizar el título universitario en tres años con un promedio arriba de 85 puntos. Otra meta sería: el $90 \%$ de los estudiantes que comienzan sus estudios en la universidad lograrán concluir sus estudios y recibir su título luego de 4 años.

Cada una de las metas serán especificadas en acciones o comportamientos específicos que tanto la dirección, el personal administrativo, los clientes y proveedores de la organización realizarán a diario en forma rutinaria o específica para cumplir las metas trazadas. Las acciones diarias de la universidad comprenden las clases frontales, la investigación, el mercadeo y publicidad de la universidad, la manutención y la limpieza diarias. Todas estas acciones son necesarias para el cumplimiento de las metas de la universidad. Volviendo al ejemplo gráfico de la pirámide, podemos inferir entonces que la porción de tiempo más amplia que ocupa la mayor superficie de la pirámide es la etapa de acción o conductas recurrentes. Estas conductas deben ser evaluadas por medio de instrumentos de medición aplicados esporádicamente y al final de cada período de actividad preestablecido.

Sintetizando el proceso, podemos apreciar el ciclo de planificación en el cual se plantea la etapa del pensamiento creativo como inicio y se deriva en forma descendiente por las distintas etapas hasta llegar a la acción concreta, para nuevamente retomar todas las etapas en forma ascendente (Ver las flechas direccionales en la Figura No. 4). Si las acciones fueron satisfactorias es porque concretizamos las metas. Si las metas fueron logradas es porque cumplimos los objetivos. Si los objetivos fueron cumplidos realizamos nuestra misión y nos acercamos a la visión deseada, tendiendo a cumplir los valores organizacionales. Entonces podremos recomenzar un nuevo proceso de planificación por medio de un nuevo pensamiento creativo.

\section{Aprendizaje (Learning)}

El proceso de aprendizaje es un continuo en forma de escalera ascendente con forma de caracol. Desde los primeros desempeños del bebé recién nacido, hasta las complicadas tareas que realiza el adulto en su vida diaria, todo es aprendizaje. Una organización que no tiene capacidad de aprender y de enseñar a sus miembros no podrá prolongar 
su vida útil. Cada director, ejecutivo, coordinador u operario en una organización debe tener en claro que su capacitación profesional es constante e interminable. A su vez, la organización misma tiene que contar con programas de adiestramiento y rumbos de planificación de carrera de sus miembros.

El capital humano en las organizaciones actuales es de primordial importancia ya que la mayor parte de los conocimientos y aptitudes están en las cabezas de los miembros de la organización y no necesariamente en las computadoras y maquinarias. La sabiduría y el estudio que la organización brinda a sus miembros son una interminable fuente motivacional. En el ejemplo de la institución universitaria el proceso de aprendizaje es incesante y de él depende la subsistencia de la organización como tal.

\section{Acabable (Achievable)}

Toda organización en el mundo acelerado y dinámico en el que se vive en el siglo XXI, tiene que aumentar la factibilidad de sus metas. Los directores y administradores que en el siglo XX se conformaban con mantener el status quo, o de generar logros moderados, se ven obligados, por el rápido ritmo del cambio, a ejecutar y lograr los objetivos organizacionales. Lo único constante en el Universo es el cambio que sostiene la teoría del cambio planificado. Por lo tanto, el lograr las metas trazadas por la organización no es toda la tarea a realizar, sino que al lograr una meta u objetivo inmediatamente se debe replantear un cambio de perspectiva que lleve a los nuevos objetivos y metas. ¿Tiene acaso, la universidad en su forma existente hoy, la factibilidad máxima de subsistir y sobrevivir durante todo el siglo XXI? ¿Qué cambios estructurales debe llevar a cabo la universidad para lograr en forma óptima sus objetivos y metas?

\section{Normas}

Las normas o patrones de conducta de las organizaciones son creadas para lograr de la mejor manera posible los objetivos de la organización, teniendo en cuenta la influencia de dichas normas en el personal de la organización y viceversa. Las normas no pueden ser establecidas unilateralmente, sino por medio de un diálogo democrático 
dentro de la organización tomando en cuenta los intereses de todos sus miembros. De esta manera, se crean modelos únicos y especialmente adaptados no solo a cada organización, sino también a cada empleado y a cada situación creada. Estas reglas de conducta se transforman en unidades básicas y típicas de conformación del Plankton organizacional. Si bien son difíciles de identificar a simple vista, influyen sobre el éxito de la organización a largo plazo y en gran escala.Las normas, así como el Plankton, pueden ser observadas fijándose en las microscópicas unidades de conducta del empleado o el director en su labor diaria o pueden verse a gran escala y con perspectiva alejándose de las unidades organizacionales para observar comprehensivamente la conducta dela organización como un todo en el marco de su entorno interorganizacional.

Las normas organizacionales son compiladas en manuales de instrucción y códigos de conducta. Pero estas normas son las normas explícitas o las normas formales, descubiertas que demarcan el funcionamiento esperado de la organización. Pero existen otro tipo de normas que no son explícitas, declaradas o escritas en compendios claros. Estas normas son conocidas como normas informales que determinan la cultura organizacional de la institución.Las normas informales se van tejiendo a través del tiempo y se pasan de generación en generación de trabajadores. Estas normas se pueden apreciar en las conductas diarias de los trabajadores y son determinantes, difíciles de cambiar y basadas sobre todo en el ejemplo personal de los rangos directivos.El éxito o fracaso de una organización puede deberse en gran manera a los postulados por las normas formales y a la definición del estilo particular de las normas informales que existen en la organización.

\section{Conocimientos}

Conocimiento es Poder ("Knowledge is Power"). En la sociedad tecnológica en la que vivimos, el saber y tener conocimientos en cualquier tema es la ventaja irrefutable en la carrera hacia el éxito y el logro de los objetivos. Las organizaciones deben tener por lo menos dos áreas de conocimientos tanto amplias como profundas. La primera área es el ramo, el mercado o el tema tecnológico en el cual la organización está insertada. La segunda área es el arte de la administración y dirección empresarial. Los conocimientos y el saber, las organizaciones, lo codifican en manuales y en bancos de datos computarizados y depositados 
hoy en tecnologías Nube ("Cloud technology").Pero, la sabiduría organizacional la llevan principalmente los directivos y empleados de la organización en sus cabezas. Por eso el capital humano debe ser cuidado, promovido y asegurado por la organización. Cada uno de los miembros de la organización funcionan como un rompecabezas, donde cada ficha tiene sus colores, su diseño y sus cualidades que aisladas no contribuyen a nada en la tarea organizacional. Combinadas armoniosamente unas a las otras, las partes del rompecabezas crean el cuadro completo (una Gestalt), permitiendo la máxima utilización de los conocimientos en general y el poder llegar al deseado objetivo.

\section{Tecnología}

El saber tecnológico(Technological Know How) de la organización es la base práctica de la organización Plankton. El secreto de la supervivencia de todo organismo vivo está en la adaptación a los cambios internos y externos. Así fue lo propuesto por las teorías del siglo $\mathrm{XX}$, esto ocurre también en toda organización que funciona como un organismo vivo. Los cambios en el siglo XXI son cada vez más rápidos y más frecuentes hasta tal punto que la naturaleza humana se dificulta en adaptarse a dichos cambios y las organizaciones se comportan como tal. El rápido progreso ha dejado atrás imperios económicos basados en tecnologías caducas y ha promovido a un nuevo éxito económico y tecnológico empresas que se crearon sobre nuevas bases tecnológicas en relativamente poco tiempo. Los adelantos tecnológicos los vemos en todas las áreas. En el campo de la ciencia y la medicina, en los instrumentos de precisión y en el uso incesante de tecnologías de informática para mejorar el nivel de vida. La dependencia tecnológica lleva a las organizaciones a poder sobrevivir en el entorno interorganizacional solamente si actualizan constantemente sus tecnologías informáticas y los

equipos de trabajo. Ésta misma dependencia puede hacer sucumbir organizaciones enteras en catástrofes naturales o mega atentados terroristas como ocurrió en el de las Torres Gemelas en New York en el año 2001. Por lo tanto, la carrera detrás de la tecnología es obligatoria para toda organización y determinante en su supervivencia. El factor humano y la tecnología directiva aplicada por sus directores es de suma importancia. Una universidad que no renueva sus recursos tecnológicos dejará de ser atractiva a nuevos estudiantes y desaparecerá en poco tiempo. 


\section{Oportunidades}

Toda organización debe marchar hacia adelante. De no hacerlo así lo más probable es que se quede en el lugar o que retroceda. Es así que el crecimiento y el desarrollo de la organización son casi constantes y obligatorios. Las organizaciones y sus miembros se encuentran en una continua búsqueda de oportunidades. Como el Plankton que parece viajar a la deriva adonde las corrientes marinas lo transporte, pero en realidad el Plankton busca el rumbo donde pueda ser de mayor utilidad a sus unidades miembros. De esta manera, cumple su función de la mejor manera posible sirviendo a sus miembros y a su entorno general. También las organizaciones buscan las oportunidades que se presentan a su paso para poder cumplir su función hacia sus miembros y en el entorno interorganizacional en el que está inserta. Oportunidades, son aquellos eventos que permiten en forma planificada generalmente, y a veces en forma espontánea y sorpresiva, aprovechar los recursos organizacionales y las situaciones creadas, que cuando se combinan, catapultan a la organización a rumbos previamente inesperados.

Las oportunidades deben ser identificadas, catalogadas y aprovechadas en su justo momento para el beneficio de la organización y de su visión. La organización debe crear en la conciencia de cada uno de sus miembros la expectativa que la oportunidad va a llegar en cualquier momento y que sus miembros deben poder identificarla. De no ser así, la oportunidad se pierde y probablemente otra organización logre aprovecharla.

\section{Natural}

La madre Naturaleza es sabia y poderosa. El correcto uso de los recursos naturales y la explotación moderada son la mejor lección que una organización puede aprender. El Plankton se ve interminable por medio de satélites desde el espacio. Los principales beneficiarios de su abundante presencia parecen ser solo las enormes ballenas azules que devoran toneladas de Plankton por día. Pero el Plankton no se termina nunca gracias a un equilibrio ecológico casi perfecto que balancea el aprovechamiento de los recursos naturales. Toda organización debe planificar en forma equilibrada la explotación de los recursos naturales (materiales, tierras, aguas, aire, minerales, caza de animales, pesca etc.) en un contexto de ecología organizacional. $\mathrm{O}$ sea, la posibilidad de integrarse en la cadena ecológica 
tratando de crear continuidad y no extinguirse a sí misma y a su alrededor. La tarea natural de la organización comienza en la administración interna de los recursos existentes, el reciclaje y el re-uso de materiales desechables y maquinarias en desuso. La planificación para la reducción de la generación de basura permite la perpetuidad de la vida útil de lo producido por la organización y de sus unidades miembros. La tendencia hacia lo natural en la organización Plankton, puede generar una tendencia a la supervivencia y a la continuidad organizacional deseada por muchos años más.

\section{Conclusión}

La teoría de la organización Plankton presentada en este trabajo propuso en forma innovadora una perspectiva distinta de los marcos teóricos conocidos y desarrollados durante el siglo XX. No cabe duda de que las teorías clásicas y las teorías emergentes son la base indiscutible de los conocimientos requeridos para una administración efectiva en las organizaciones creadas en el siglo pasado. Pero, los grandes y acelerados cambios percibidos desde principios del siglo XXI nos exigen nuevas propuestas que traten de enmarcar los nuevos desafíos organizacionales a los que nos enfrentamos.

Podemos demarcar las conclusiones en los siguientes puntos:

1. Existe hoy la necesidad de conocimientos amplios y multidisciplinarios de aquellos profesionales que pretenden comprender la conducta organizacional en el marco interorganizacional del siglo XXI para un mejor desempleo de ellos. La información es el capital con el que cuentan y la posibilidad de manipular dicha información es el desafío al que se enfrentan los directores de las organizaciones de servicios humanos. El bagaje teórico del siglo $\mathrm{XX}$, compuesto por las teorías clásicas y emergentes, es la base de la propuesta, que combinado con las apreciaciones de la nueva teoría de la organización Plankton aquí presentada pueden otorgar una innovadora y práctica perspectiva organizacional.

2. El acrónimo Plankton como título conductor de la teoría propuesta responde a la creciente necesidad de enunciar conceptos lo suficientemente dinámicos y creativos tanto así como actuales y atractivos. En la sociedad humana de hoy, los cambios suceden a un ritmo extremadamente acelerado y a veces casi inalcanzable. 
La interminable creatividad humana nos ayuda no sólo a proponer como solución la adaptación a los cambios sino también contribuye a seguir ininterrumpidamente el descubrimiento de nuevas tecnologías y nuevas tendencias. El mercadeo de bienes y servicios depende de la calidad ofrecida tanto así como de la forma en que ellos son presentados. Por eso, la teoría de la organización Plankton plantea una propuesta innovadora utilizando un concepto recurrente en el mundo que es presentado de acuerdo con las pautas requeridas actualmente y establecidas en la sociedad del siglo XXI.

3. La teoría de la organización Plankton utiliza ocho conceptos definidos como básicos para poder abordar la teoría: planificación, aprendizaje, acabable, normas, conocimientos, tecnología, oportunidad y natural. El Plankton como unidad básica de la vida en la Tierra se proyecta metafóricamente como unidad básica de la vida de la organización. Sin poder identificar y promover el Plankton organizacional, la organización toda corre peligro. El abordaje serio y profesional de cada uno de los conceptos es esencial para la supervivencia de la organización. El trabajo presentó ejemplos concretos de cómo pueden ser abordados los ocho conceptos básicos.

4. La investigación de las organizaciones en el siglo XXI y el desarrollo de nuevos conceptos teóricos con base en dichas investigaciones, deben estar basados en una combinación equilibrada del trabajo académico en las universidades y de la práctica sustancial de campo. La teoría de la organización Plankton se forjó durante años por medio de esta doble experiencia llevada a cabo por el autor. La práctica de asesoría y consultoría organizacional en instituciones con y sin fines de lucro, privadas y públicas otorgó la experiencia de campo necesaria. El trabajo académico de investigación, enseñanza y aprendizaje en universidades proporcionó el marco teórico. Esta combinación debe ser sugerida como requisito para futuros desarrollos de nuevas teorías de la organización. La teoría de la organización Plankton trató humildemente de presentar una alternativa de pensamiento conceptual que nos puede ayudar en la incesante tarea de búsqueda de la brecha profesional adecuada y adaptable al mundo de hoy. 


\section{Referencias bibliográficas}

Bennis W.G., Benne K. D., Chin, R., \& Corey, K.E. (Eds). (1961).The Planning of Change New York: Holt, Rinehart \& Winston.

Follet M.P. (1924). Creative Experience. New York: Longmans, Green.

Gulick, L. \& Urwick, L. (1937). Papers on the Science of Administration. New York: Institute of Public Administration.

Hersey, P. \& Blanchard, K.H. (1977). Management off Organizational Behavior: Utilizing Human Resources. Englewood Cliffs, N.J.: Prentice Hall.

Lewin, K. (1948). Resolving Social Conflicts: Selected Papers on Group Dynamics. New York: Harper \& Row.

Maslow, A. (1954). Motivation and Personality. New York: Harper \& Row.

Merton, R. K. (1968). Social Theory and Social Structure. Ney York: Free Press.

Parson, T. (1949). The Structure of Social Action. Glencoe Ill.:Free Press.

Silverman, D. (1971). The Theory of Organizations: A Sociological Framework. New York: Basic Books.

Simon, H. (1960). The New Science of Management Decisions. New York: Harper \& Row.

Taylor, F.W. (1945). Scientific Management. New York: Harper \& Row.

Thomson, J.D. \& McEwen, W. (1958). Organizational Goals and Environment: Goal Setting as an Interaction Process. American Sociological Review, 23 (1), 23-31.

Von Bertalanffy, L. (1968). General System Theory: Foundations, Development, Applications. New York: Braziller.

Von Neumann, J. \& Morgenstern, O. (1944). Theory of Game \& Economic Behavior. Princeton, N.J.: Princeton University Press.

Weber, M. (1945). The Theory of Social and Economic Organizations. Glencoe, Ill. Free Press.

Weiner, M.E. (1990). Human Services Management: Analysis and Applications. The Dorsey Press, Homewood, Illinois.

Woodward, J. (1958). Management and Technology. London, H.S.M.O. 